\title{
Design of a "beetle-type" atomic force microscope using the beam deflection technique
}

\author{
B. Gasser, A. Menck, H. Brune, and K. Kern \\ Institut de Physique Expérimentale, Ecole Polytechnique Fédérale Lausanne, \\ CH-1015 Lausanne, Switzerland
}

(Received 27 November 1995; accepted for publication 23 January 1996)

\begin{abstract}
In the present article we describe a new setup for an atomic force microscope in the beetle-type geometry. The microscope consists of a compact head standing on three piezo legs with a fourth central piezo carrying the cantilever tip. We use the laser beam deflection method to detect the deflection of the cantilever. All optical components are integrated into the microscope head which has a diameter of $40 \mathrm{~mm}$. This compactness results in a high mechanical stability, while the adjustment of the optical pathway is still easy to handle. The microscope can be used in UHV and in air. Measurements on $\mathrm{KBr}(100)$ in air show the capability of the microscope to obtain a resolution up to atomic corrugations. (C) 1996 American Institute of Physics. [S0034-6748(96)01105-9]
\end{abstract}

\section{INTRODUCTION}

The atomic force microscope (AFM) provides highresolution images of force contours at or close to a sample surface. The most important properties of the AFM are the capability to image insulating surfaces and the ability to investigate phenomena associated with various forces, such as adhesion, ${ }^{1}$ surface charges, ${ }^{2}$ magnetism, ${ }^{3}$ and friction. ${ }^{4} \mathrm{~A}$ variety of different types, working in ambient conditions, ${ }^{5}$ liquids, ${ }^{6}$ or ultrahigh vacuum (UHV) ${ }^{7}$ and several different methods for detection of the force, i.e., the cantilever's deflection, have been described. ${ }^{8}$

In this article we present a new design for an atomic force microscope based on the so-called "beetle-type" scanning tunneling microscope (STM). This STM design was first introduced by Besocke ${ }^{9}$ and has later been extended by an inertial coarse approach. ${ }^{10}$

Driven by the motivation to design a variable temperature and UHV-compatible AFM we chose the beetle-type design since it compensates to first-order thermal drift of the tip-to-sample distance. The setup presented here is rather compact which yields high mechanical eigenfrequencies and thus a low sensitivity to external vibrations. In addition small distances of the reflected beam result in low drifts of the force setpoint. The further advantage for us was compatibility to the design of our variable temperature STMs. ${ }^{11}$

Wiechers $^{12}$ have recently reported the construction of a beetle-type AFM where the geometry is such that the sample lies on three piezo legs and the microscope body is at rest, the same as originally designed by Besocke. In our setup, a very compact scan head stands with its three outer piezo legs on the sample holder. This upside-down geometry has been chosen since for variable temperature applications it is most appropriate to rigidly mount the sample onto a cryogenic manipulator. ${ }^{11,13}$

We have chosen the beam deflection method for this microscope as it offers several advantages. It provides the possibility of detecting simultaneously normal and lateral forces. ${ }^{14,15}$ The sensitivity of the method is high enough to resolve the periodicity of the atomic lattice ${ }^{16}$ and its proper- ties are well characterized due to its widespread application. All optical components necessary for the beam deflection method together with mechanical adjustment facilities have been integrated into a scan head of $40 \mathrm{~mm}$ diameter. We show first results obtained under ambient conditions. They demonstrate a high mechanical stability and reliable operation of this new compact beetle-type AFM.

\section{THE AFM HEAD}

A schematic drawing and a photograph of the beetletype AFM are shown in Fig. 1. The microscope stands with ruby balls, which are glued to the ends of the three outer piezos, on a triple helix sample holder. The sample holder provides the typical ramps for coarse inertial approach and is made of anodized $\mathrm{Al}$ in order to provide hard surfaces for the gliding motion of the ruby balls (for UHV, e.g., polished Mo can be used). The cantilever is carried by the central piezo.

The microscope head comprises four disks. Each of them carries one optical component of the beam deflection technique. Their lateral positions with respect to each other are well defined by leaf springs that connect successive disks. Their respective distance and tilt can be adjusted via three screws which are under preload by the leaf springs. The uppermost disk (1) carries the light source. For operation in air it is most appropriate to mount the laser diode (e.g., HL7806G, $P_{0}=5 \mathrm{~mW}, \lambda=780 \mathrm{~nm}$, HITACHI) directly onto the disk of the AFM head (see the photograph). For UHV application, the laser diode must be placed outside the vacuum chamber since it would likely be destroyed during bake out. In this case a single-mode fiber is used to transmit the light to the AFM head. Its end is the light source ( $\phi 5$ $\mu \mathrm{m})$. For test in air we used a "pigtailed" laser diode (diode HITACHI HL7812G, $\lambda=780 \mathrm{~nm}$, pigtailed by Seastar Optics Inc., Canada, power at the fiber end $3 \mathrm{~mW}$ ). The fiber is typically glued to a UHV feedthrough and therefore attached to the chamber. For practical reasons it is convenient to be able to disconnect the microscope from the chamber. Therefore we chose an FC/PC connector between the light emitting fiber end and the uppermost disk of the AFM (see draw- 

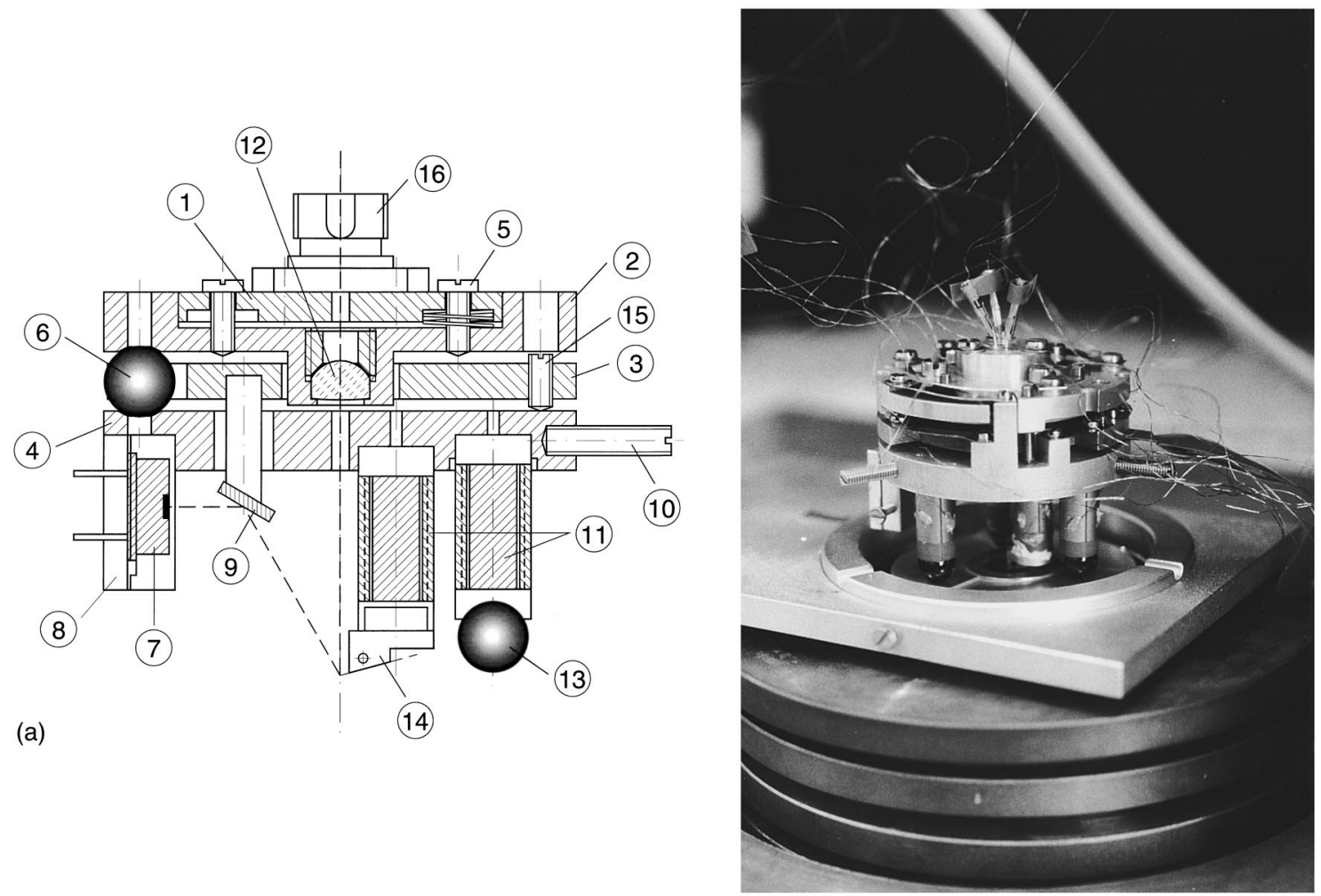

(b)

FIG. 1. (a) Schematic drawing of the AFM. (1) Uppermost disk, carrying the FC/PC connector (16) of the fiber of a pigtailed laser diode; (2) disk to which the lens (12) is mounted; (3) intermediate disk carrying the mirror (9), tilting it by means of the screws (15) adjusts the reflected laser beam on the position sensitive detector [PSD (7)]; (4) disk to which piezo tubes, cantilever, and PSD are mounted; (5) screw serving for adjustment of the focus; (8) housing of the PSD; (10) support screws for lifting the whole AFM; (11) piezo tubes, (13) ruby balls; (14) cantilever support. (b) Photograph of the AFM. The AFM stands on the sample holder which is mounted on a stack of copper plates. In this configuration used for operation in air, the light source is a laser diode directly mounted to the uppermost disk (1).

ing for this version of the disk). This connection guarantees a well-defined lateral and vertical position of the fiber end so that disconnecting and reconnecting of the fiber required only minor readjustment of the optics.

In front of the light source there is a lens $(f=5 \mathrm{~mm}$, "best form," Melles Griot) mounted rigidly onto the second disk (2). It generates an image of the light source four times bigger onto the backside of the cantilever. The diameter of the resulting spot can be adjusted by varying the distance of the light source, i.e., disk 1, with respect to this lens, i.e., disk 2, by turning the three screws [focus (5)]. The smallest obtainable spot diameter is about $20 \mu \mathrm{m}$, which is less than the width of commercially available cantilevers.

The position where the laser spot hits the cantilever can be adjusted by tilting the whole assembly comprising light source and lens, i.e., disk 2, with respect to disk 4, to which the piezos and the cantilever are mounted. This can be achieved by two screws (not visible in Fig. 1) which define a plane together with the stainless-steel ball (6). Finally the beam, after being reflected from the cantilever, must be positioned onto the center of the four-quadrant photodiode (SFH244S, Siemens) at zero force. This adjustment has been divided into a coarse and a fine part in order to have the necessary precision and to be able to use several levers on a multilever chip without moving it in its holder. The detector (7) can be moved by $\pm 1 \mathrm{~mm}$ in $x$ and $y$ before it is clamped on the brim of its housing (TO39) into a chassis (8) mounted to disk 4. Once the coarse position of the detector has been fixed, a fine adjustment can be done by tilting the intermediate disk (3) of the microscope which carries a mirror (9) that reflects the beam onto the detector. Tilt of the mirror in two orthogonal directions enables one to adjust both normal and lateral forces. A further possibility for coarse adjustment of the normal force consists of varying the height of disk 3 .

This way of adjusting the optical components has proven to make the different adjustment tasks, as, e.g., focusing, hitting the backside of the lever, and guiding the reflected beam onto the middle of the position sensitive detector, sufficiently independent. This allows a stepwise refinement to quickly maximize the performance of the beam deflection method for each lever. The construction of the mechanical components and their connection by leaf springs turned out to be sufficiently stiff to avoid mechanical vibrations below the first eigenmode of the piezo tubes (11) (see below). For sample and tip exchange the whole microscope can be lifted from the sample holder on three screws (10) radial fixed to disk 2.

We use microfabricated cantilevers made of $\mathrm{Si}_{3} \mathrm{Ni}_{4}$ (STMS-06AU, Park Scientific Instruments) with typical force constants of $0.01-0.50 \mathrm{~N} / \mathrm{m}$. The cantilever is attached by means of a small spring ( $W$ wire) to a small support piece glued to the end of the inner piezo tube. The angle of the cantilever with respect to the horizontal is $15^{\circ}$.

The outer electrodes of the piezo tubes (EBL1, Staveley 
Sensors) are segmented into quarters. The three outer piezo legs are used for inertial coarse approach and lateral displacement. ${ }^{10}$ The outer piezos can also be used for scanning which, however, limits the scan speed due to the inertia of the microscope body. Therefore we usually prefer to scan the tip with the inner piezo. This, however, causes virtual force changes due to the variation of the lever's angle due to bending of the piezo tube. In addition, since the probe is mounted off axis with respect to the piezo tube, the height change during the scanning movement has to be compensated by the feedback and causes a tilt of the image. One can largely get rid of both of these effects by bending the piezo tube in an $\mathrm{S}$ shape, which can be achieved by subdividing the four electrodes into two pieces each, to which opposite voltages are applied. ${ }^{17,18}$ The coupling of the fast scanning movement, which we chose to be perpendicular to the plane of the beam, to the force signal has been found to be negligible. The vertical displacement of the cantilever is accomplished via the inner electrode of the central piezo tube.

The sample holder is fixed on the upper plate of a conventional stack of copper plates connected to each other with viton U's which act as soft, damped springs. This stack efficiently suppresses external vibrations at frequencies higher than the eigenmodes of the individual plates $(30-50 \mathrm{~Hz})$. In order to damp vibrations of the building $(10-50 \mathrm{~Hz})$, where the transmission of the stack is high, the whole stack stands on a massive stone plate which itself resides on air suspended laser feet. The mechanical stiffness of the microscope, however, is sufficiently high to allow reliable operation $\left(\Delta z_{\text {peak to peak }} \leqslant 0.2 \AA\right)$ without the air suspension of the table.

\section{ELECTRONICS}

We used a commercial power supply for the laser diode (Type LD-2000, Seastar Optics Inc.) which we operate in the constant current mode. The current signal from the four quadrants of the position sensitive detector is measured with four $I / V$ converters located close to the microscope (conversion $10^{5} \Omega$, bandwidth $30 \mathrm{kHz}$ ). The vertical and horizontal difference of the signals obtained from the four quadrants are proportional to the normal and lateral force, respectively. For adjustment reasons also the sum of all quadrants, i.e., a signal proportional to the total intensity shining on the detector, is provided. The difference signal for the normal force is compared to a setpoint. This error signal is then amplified with a gain of $0-1000$ (speed of the feedback loop) and subsequently integrated $(4.82 \mathrm{k} \Omega, 3.3 \mu \mathrm{F})$. The output of the integrator is post amplified $( \pm 220 \mathrm{~V}$ amplifier, using hybrid circuits PA88A, APEX Microtechnology Corporation, Arizona) and then connected to the $z$ electrode of the central piezo tube. The resulting vertical range is $1.32 \mu \mathrm{m}$.

The scanning movement and data acquisition are done via commercially available electronics. ${ }^{19}$ To increase the scan range we amplified the $x$ and $y$ signals which results in a maximum scan range of $3 \mu \mathrm{m}$ at $\pm 220 \mathrm{~V}$ (high voltage amplifiers identical to those used for $z$ ). A PC serves to record the $z$ signal. Due to the limited resolution of the ADC, this is mostly done in the often called "differential mode"
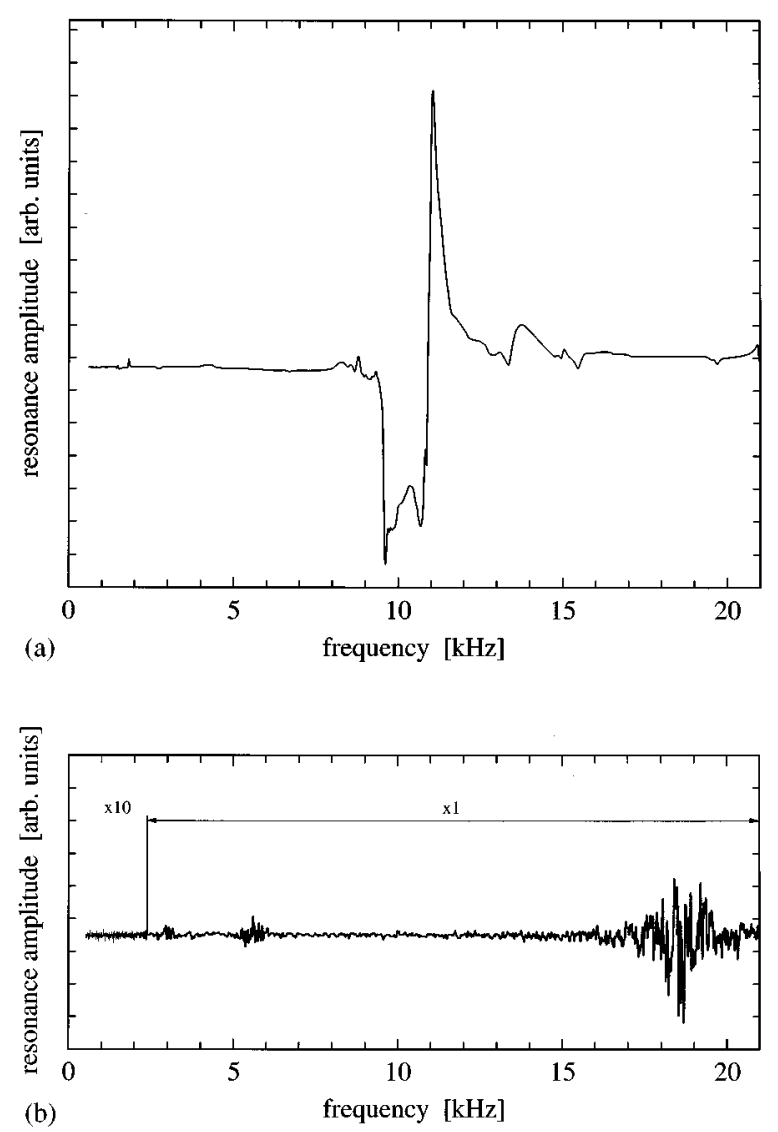

FIG. 2. Noise spectra in the range of $200 \mathrm{~Hz}-20 \mathrm{kHz}$. (a) Excitation at the inner piezo tube and mechanical response at one of the outer piezos when the microscope is placed upon the sample holder. (b) Noise spectrum of the force signal taken with the cantilever being far away from the sample.

where a $30 \mathrm{~Hz}(330 \mathrm{~Hz})$ highpass filter of first order is put in front of the ADC. ${ }^{19}$ Images recorded this way appear as if light shines on the surface from the left.

\section{MECHANICAL STABILITY}

Figure 2 represents typical noise spectra taken with the AFM placed upon the sample holder. In Fig. 2(a) we show the vibration spectrum recorded with a lock-in amplifier connected to one of the electrodes of the outer piezos while a bending motion has been excited by the reference output on the inner piezo tube (a sinus with $10 \mathrm{~V}$ amplitude was used). In this way, the microscope head is vibrated due to the inertia of the inner piezo and the tip holder and its vibration amplitude is measured as a piezoelectric voltage at one of the legs. The lowest considerable eigenfrequency is at $9.6 \mathrm{kHz}$ and thus satisfactorily high. This shows that the size of our microscope is a good compromise between, on the one hand, the size required for all optical components and that the optical adjustment is easy to operate, and, on the other hand, the ultimate minimization to obtain mechanical stiffness.

In order to identify the part of the mechanics responsible for this lowest eigenmode, we performed calculations for the bending modes of the disks. These, however, lie far above 10 $\mathrm{kHz}$. In contrast, the lowest eigenmode of the piezo tubes themselves seems to be within the frequency range of interest. 
A flexible shaft of no mass which is clamped on one side, i.e., a cantilever beam, has on its free end a spring constant of $k=3 E I / l^{3}$, with $E$ being the modulus of elasticity, $I$ the surface moment of inertia, and $l$ the lever's length (page 158 of Ref. 20). Thus the fundamental mode of a weightless cantilever beam with a mass $M$ attached to its free end is $\omega_{1}=\sqrt{3} \sqrt{E I / M l^{3}}$. That of a cantilever of mass $m$, on the other hand, is $\omega_{2}=3.515 \sqrt{E I / m^{3}}$ (p. 223, Ref. 20). Due to Dunkerley's formula (p. 302, Ref. 20) the frequency of a cantilever beam of mass $m$ with a mass $M$ attached to its free end can be written as

$$
\omega^{2}=\frac{\omega_{1}^{2} \omega_{2}^{2}}{\omega_{1}^{2}+\omega_{2}^{2}}=\frac{I E}{l^{3}} \frac{3 \times 3.515^{2}}{3 m+3.515^{2} M} .
$$

The moment of inertia (surface) of a tube upon bending is $I=\pi / 4\left(R^{4}-r^{4}\right)$, where $R$ and $r$ are the outer and inner radius, respectively.

With the dimensions of our piezo tubes $\left(R=0.125^{\prime \prime}\right.$, $r=0.105^{\prime \prime}, l=0.61^{\prime \prime}, E=8.1 \times 10^{10} \mathrm{~N} / \mathrm{m}^{2}$, and a density of $7.5 \times 10^{3} \mathrm{~kg} / \mathrm{m}^{3}$ ) and a mass at the piezo end of $M=0.33 \mathrm{~g}$, corresponding to that of the tip holder, we obtain $f=10.6$ $\mathrm{kHz}$. This is close to the lowest eigenmode we measured, which is thus very likely due to a resonance of the inner piezo. This interpretation is supported by the fact that we measure a slightly lower fundamental mode when the microscope is lifted from the sample holder. Then the three legs are free to vibrate at their ends to which the ruby balls are glued. Since the mass of the ruby balls is increased with respect to that of the tip holder, this is expected to give rise to a slightly lower eigenfrequency as observed.

In order to characterize the mechanical stability of the positions of the optical components and the coupling of external vibrations to the cantilever, we measured the noise spectrum of the force signal. The AFM tip was far away from the sample and the only excitation was external noiselike sound and vibrations of the building. This spectrum [Fig. 2(b)] is flat apart of the resonance of the cantilever at about $16 \mathrm{kHz}$, which indicates that external noise sources are damped efficiently by the setup. The fundamental mode of the piezo tubes is not excited in this mode. As expected this changes when the feedback is operating, there this fundamental mode limits the speed of the feedback loop to below $10 \mathrm{kHz}$.

\section{RESULTS}

One example that characterizes the performance of the AFM is shown in Figs. 3 and 4. They show typical images of the $\operatorname{KBr}(100)$ cleavage plane. Figure 3 shows a large scale constant force image. Monatomic steps can clearly be resolved they are $3.3 \AA$ in height. In the center of the image screw dislocations emerge, which are the starting points of curved steps. As the surface is subjected to stress during the cleavage, the appearance of such defects is typical.

Figure 4 shows one screw dislocation at higher magnification. The apparent width of the step is about $10 \mathrm{~nm}$, which is due to the finite radius of curvature of the tip and also a rough estimation of the minimum distance to distinguish subsequent monatomic steps. A detail taken on the terrace is

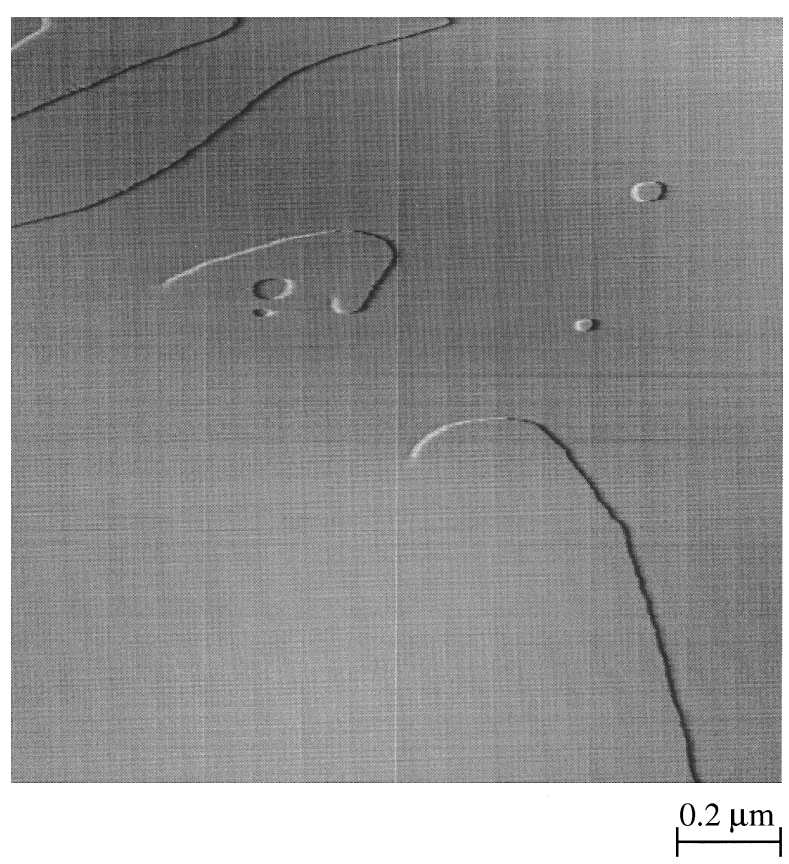

FIG. 3. $1.5 \mu \mathrm{m} \times 1.5 \mu \mathrm{m}$ image of a $\mathrm{KBr}(100)$ plane after cleavage in air. The image is taken in the differential mode; it appears as if light shined on the surface from the left.

reproduced in the inset in Fig. 4. The atomic rows of the $\mathrm{KBr}$ lattice are clearly resolved; the resolution perpendicular to these rows is less prominent. Nevertheless, the square lattice, which is typically denoted to the bromine ions ${ }^{21}$ due to their larger ionic radius, can clearly be resolved.

The images were taken at an attractive force of several $\mathrm{nN}$. The net force acting on the tip must be much

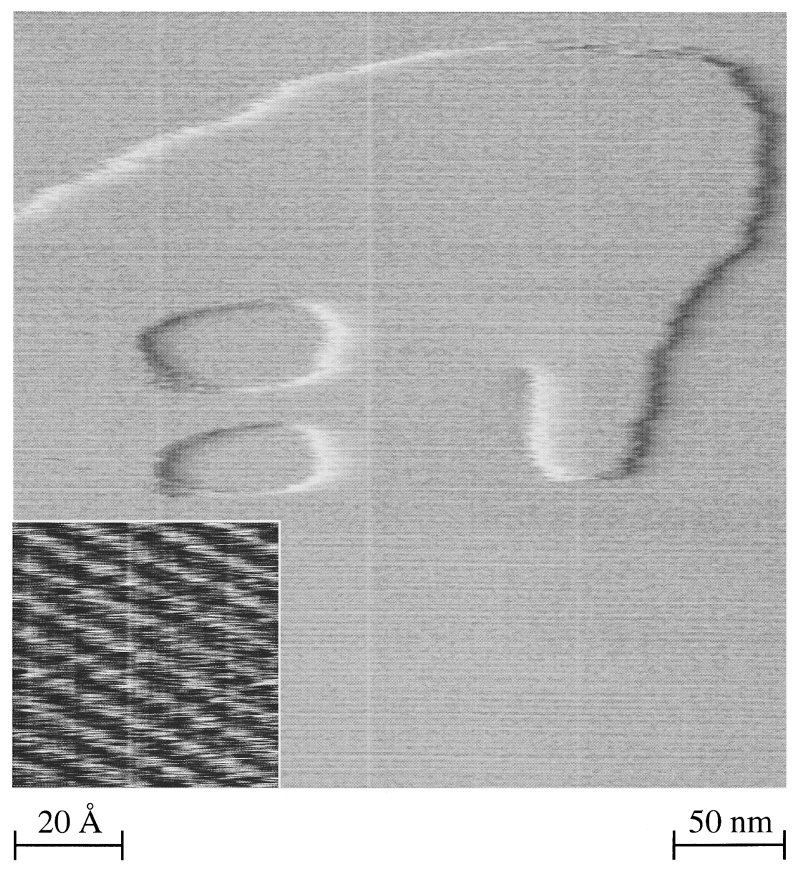

FIG. 4. $350 \mathrm{~nm} \times 350 \mathrm{~nm}$ section of previous image. The inset shows the atomically resolved (100) lattice of the $\mathrm{KBr}$ surface $(50 \AA \times 50 \AA)$. Images taken in the differential mode. 
higher, because force-distance curves yield an adhesion force of $30 \mathrm{nN}$. Thus, images of this type cannot be used as a proof of true lateral resolution, as forces are much higher than one atom can withstand. The resolution of the lattice is due to a so-called "phase-locked multitip.,"22 The AFM routinely yielded atomic resolution also on substrates with lower corrugation amplitudes as, e.g., HOPG $\left(\Delta z_{\text {typ. }}=0.2 \AA\right)$. Therefore we estimate the vertical stability of the beetle-type AFM to be less than $0.2 \AA$ peak-to-peak at the bandwidth of 10 $\mathrm{kHz}$.

\section{DISCUSSION}

We have constructed a compact AFM in beetle-type geometry. It operates with the beam deflection technique to detect the deflection of the cantilever. All optical components and their adjustments are integrated into a small microscope head. Due to its compactness the mechanical stability is high. Its first fundamental mode is found at $9.6 \mathrm{kHz}$ due to the first bending resonance of the inner piezo tube. External noise is efficiently damped by a conventional stack of copper plates with viton spacers, so that the noise level in the bandwidth of the measurement $(10 \mathrm{kHz})$ could be estimated to be less than $0.2 \AA$ peak-to-peak. This is sufficiently low to demonstrate resolution of the atomic lattice, e.g., on $\mathrm{KBr}(100)$. The microscope is fully compatible to variable temperature STMs and can also be operated in UHV.

Note added in proof: After this manuscript had been accepted we became aware of a paper by Q. Dai et al. [Rev. Sci. Instrum. 66, 5266 (1995)] that describes a "beetle-type", UHV AFM which is also suited for variable temperature operation.

\section{ACKNOWLEDGMENTS}

The authors gratefully acknowledge fruitful discussions with Jochen Wiechers, Nancy Burnham, and Giovanni Dietler.

${ }^{1}$ N. A. Burnham and R. J. Colton, J. Vac. Sci. Technol. A 7, 2906 (1989).

${ }^{2}$ C. Schönberger and S. F. Alvarado, Phys. Rev. Lett. 65, 3162 (1990).

${ }^{3}$ Y. Martin and H. K. Wickramasinghe, Appl. Phys. Lett. 50, 1455 (1987).

${ }^{4}$ C. M. Mate, G. M. McClelland, R. Erlandsson, and S. Chiang, Phys. Rev. Lett. 59, 1942 (1987).

${ }^{5}$ G. Binnig, C. F. Quate, and C. Gerber, Phys. Rev. Lett. 56, 930 (1986).

${ }^{6}$ S. Gould, O. Marti, B. Drake, L. Hellemans, C. E. Bracker, P. K. Hansma, N. L. Keder, M. M. Eddy, and G. D. Stucky, Nature 332, 332 (1988).

${ }^{7}$ G. Meyer and N. M. Amer, Appl. Phys. Lett. 53, 1045 (1988).

${ }^{8}$ E. Meyer, Prog. Surf. Sci. 41/1, 3 (1992)

${ }^{9}$ K. Besocke, Surf. Sci. 181, 145 (1987).

${ }^{10}$ J. Frohn, J. F. Wolf, K. Besocke, and M. Teske, Rev. Sci. Instrum. 60, 1200 (1989)

${ }^{11}$ H. Brune, H. Röder, C. Romainczyk, C. Boragno, and K. Kern, Appl. Phys. A 60, 167 (1995).

${ }^{12}$ J. Wiechers, Ph.D. thesis, München (1993).

${ }^{13}$ M. Bott, T. Michely, and G. Comsa, Rev. Sci. Instrum. 66, 4135 (1995).

${ }^{14}$ O. Marti, J. Colchero, and J. Mlynek, Nanotechnology 1, 141 (1990).

${ }^{15}$ G. Meyer and N. M. Amer, Appl. Phys. Lett. 57, 2089 (1990).

${ }^{16}$ G. Meyer and N. M. Amer, Appl. Phys. Lett. 56, 2100 (1990).

${ }^{17}$ M. Hipp, H. Bielefeldt, J. Colchero, O. Marti, and J. Mlynek, Ultramicroscopy 42-44, 1498 (1992).

${ }^{18} \mathrm{U}$. Dürig (private communication).

${ }^{19}$ Formerly Besocke delta phi GmbH, now distributed by Carl Zeiss Jena.

${ }^{20} \mathrm{~W}$. T. Thomson, Theory of Vibrations with Applications (Allen and Unwin, London, Sydney, Wellington, 1988).

${ }^{21}$ F. J. Giessibl and G. Binnig, Ultramicroscopy 42-44, 281 (1992).

${ }^{22}$ F. J. Giessibl, Jpn. J. Appl. Phys. 33, 3726 (1994). 